\title{
Postnatal Clubs for Integrated Postnatal Care in Johannesburg, South Africa: A Qualitative Assessment of Implementation
}

Ndinda Makina-Zimalirana ( $\square$ makinan@anovahealth.co.za )

Anova Health Institute

Jackie Dunlop

Anova Health Institute

Anele Jiyane

Anova Health Institute

Sophia Marie Bartels

University of North Carolina at Chapel Hill Louis Round Wilson Special Collections Library: The University of North Carolina at Chapel Hill

Helen Struthers

Anova Health Institute

James Mclntyre

Anova Health Institute

Kate Rees

Anova Health Institute

Research

Keywords: Antiretroviral therapy, Differentiated service delivery, HIV, Health providers, Postnatal clubs, South Africa.

Posted Date: January 31st, 2022

DOI: https://doi.org/10.21203/rs.3.rs-1220412/v1

License: (c) (i) This work is licensed under a Creative Commons Attribution 4.0 International License. Read Full License 


\section{Abstract \\ Background}

South Africa has reported challenges in retaining women in Prevention of Mother-to-Child Transmission of HIV (PMTCT) programs postnatally. Due to the success of PMTCT in the antenatal period, proportionally more infant transmissions now occur after delivery. The Médecins sans Frontières (MSF) Postnatal Club (PNC) model allows for integrated postnatal care and support. Anova Health Institute implemented the model in primary health facilities in Johannesburg as part of a planned national scaleup. We aimed to assess the implementation of these PNCs.

\section{Methods}

We used the RE-AIM (Reach, Adoption, Implementation, Maintenance) framework to assess implementation success and explore factors influencing implementation. In-depth interviews were conducted with 15 PNC staff, both clinicians and lay counsellors, using convenience sampling, from 12 facilities in Johannesburg. Data were analysed thematically using the RE-AIM framework.

\section{Results}

PNC were perceived to have many benefits for postnatal clients and their infants: providers reported reduced waiting times, reduced number of clinic visits and that PNC provided clients with a space to form cohesive group dynamics thereby contributing to retention and adherence to antiretroviral therapy. However, it was found that lacking resources (e.g., space, medical equipment, staff) negatively impacted reach, implementation and sustainability. At times the PNC model was altered to accommodate the availability of resources (e.g., counselling mothers individually). Additionally, providers expressed concerns about lack of stakeholder adoption and emphasized the importance of involving facility leadership for successful integration of the model into routine primary healthcare.

\section{Conclusion}

Our study found incomplete implementation of PNC in most of the participating facilities attributed to lack of resources and stakeholder buy-in. This underscores the need for increased support at management level to ensure sustainability. Effective collaboration between all stakeholders would allow better use of existing resources. Further studies are needed to evaluate whether all components of the model need to be implemented fully to ensure optimal outcomes, and to identify implementation strategies to facilitate scale-up.

\section{Contribution To The Literature}


- Considering that most interventions are implemented in resource constrained settings in lower- and middle-income countries, it is imperative to assess implementation especially in the initial phases. Such assessments can identify intervention elements that are impractical to implement given available resources. Our study identified the group element of the PNC model to be challenging to implement and proposed further research on how the model can be implemented with adjustments.

- This study adds to the limited literature on what has enabled, and challenged, the integration of HIV and Maternal and Child Health care through differentiated care models from the perspectives of health providers.

- The study design may inform researchers and interventionists in other similar settings on the use of the RE-AIM framework in their implementation efforts.

\section{Background}

Antenatal Prevention of Mother-to-Child Transmission (PMTCT) services have been successfully implemented in South Africa, leading to a substantial reduction in vertical HIV transmission (1). However, it has been difficult to retain women in PMTCT programs after they have given birth; with a review of large studies in a number of lower and middle countries, including South Africa, finding that $76 \%$ of pregnant women adhered to antiretroviral therapy (ART) during pregnancy, but only $53 \%$ did so postbirth(2) Overall, with fewer children becoming HIV-infected through vertical transmission, more than half of infant HIV-infections now occur in the postnatal period through breastfeeding rather than during pregnancy or labour (3). In order to address postnatal MTCT, carefully designed interventions need to target retaining women in care postnatally. One way of approaching this would be to provide integrated care to the mother-infant pair (MIP). There is a broad consensus on the value of integrating HIV and reproductive health services, particularly in regions of the world with generalised HIV/AIDS epidemics and high reproductive morbidity $(4,5)$

Postnatal Clubs (PNCs) are an integrated, evidence-based model for comprehensive HIV and Maternal and Child Health care for postpartum women living with HIV and their infants. PNCs were originally designed and implemented in the Western Cape Province by Médecins sans Frontières (MSF) and have shown promising results in this setting (6). Empirical evidence demonstrates PNC models are more effective than standard of care in retaining clients in care and that the integration of services, of which PNC is built on, has the potential to improve uptake of either reproductive health services, HIV services, or both (7). Results from pilot programs suggest that PNC are acceptable and feasible and trigger knowledge acquisition, behavior change and offer peer support $(8,9)$

However, translating this success to scale requires effective implementation of the model to achieve health impact. The shift from providing vertical services to the integration of postnatal HIV treatment services into maternal, neonatal and child health care is a complex transformation that demands significant adjustments in major aspects of health care organisation including providers' workloads (10), and infrastructural and logistic considerations (local service policies, physical space, information systems, equipment, drugs and other medical supplies) (5). These factors can be analytically studied 
through Implementation Science (IS), which is the scientific practice of identifying, testing and scaling up effective interventions with high quality, fidelity and efficiency $(11,12)$.

Implementation Science is increasingly relevant within health programs to ensure that innovations and tools reach populations in need and are appropriately adapted to the local context while maintaining core elements of proven strategies. Implementation Science frameworks like the Reach, Effectiveness, Adoption, Implementation, and Maintenance (RE-AIM) have been pioneered to guide interventionists to identify program components that will "improve the sustainable adoption and implementation of effective, evidence-based health promotion programs" (12-14). Holtrop 2018 et al demonstrated the value of qualitative measures in RE-AIM (and other planning and evaluation approaches) to address questions regarding the scalability and sustainability of innovations (14). The paper demonstrated that qualitative methods help us understand how and why results on various individual RE-AIM dimensions, or patterns of results across dimensions (e.g. high reach and low effectiveness) occur.

The RE-AIM was chosen to evaluate PNC implementation because RE-AIM dimensions allow for the investigation of the degree to which an intervention can be adopted consistently and delivered in a sustained manner, reach large numbers of people and produce replicable behaviour changes (15) RE-AIM is a widely accepted framework used to assess the feasibility, quality, and public health impact of a health intervention (14) Additionally, the framework offers flexibility to address different public health concerns in a practical manner understandable by practitioners and policy makers (15).

While the PNC model has been adopted nationally in South Africa, it is yet to be implemented at scale. As a result, there is a need to understand how the PNC model is implemented to inform scale up to achieve the intended impact. To this end, we conducted a qualitative assessment of the implementation of postnatal clubs supported by the Anova/APACE program in Johannesburg. We utilised the RE-AIM framework for this study to provide an understanding of a range of factors influencing implementation outcomes.

\section{Methods}

\section{Postnatal Clubs Model}

The MSF Postnatal Club model was piloted by MSF in Khayelitsha, Cape Town, South Africa. The PNC model integrates PMTCT and maternal and child health $(\mathrm{MCH})$ services during the postnatal period. It includes screening for postpartum mental health disorders and group educational components, and is delivered to postpartum women and their infants during their 18-month postnatal period. Facility and community-based lay health care workers coordinate the running of clubs, provide peer support and health education on PMTCT and Early Childhood Development (ECD).

The four pillars of PNC are: 
1. Adult ART adherence club model which includes group peer support and adherence counselling for the mothers,

2. Early childhood development activities including mental health screening and breastfeeding support for mothers,

3. Integration of maternal and child health which encourages a comprehensive one-stop shop for the clinical care for mother and baby at each session including pre-packaged ART, and

4. Integration of HIV and non-HIV care, for both mother and baby

Figure 1: PNC components

Evidence demonstrates that the PNC model shown in figure 1 is more effective than standard of care:

1. in retaining and virologically suppressing mothers in care,

2. ensuring HIV-exposed infants complete their HIV testing schedule and remain HIV negative, and

3. ensuring HIV-exposed infants are fully immunised and receive other essential child health interventions.

\section{Study setting}

Johannesburg, one of 52 health districts in South Africa, has a population of about 5.5 million, almost $10 \%$ of the country's population, and is the country's largest Metropolitan District. The health district has over 600,000 people living with HIV (PLHIV), more than any other city worldwide (16). Johannesburg is one of 27 health districts prioritised for HIV interventions by the US-funded President's Emergency Plan for AIDS Relief (PEPFAR).

Anova is PEPFAR funded through USAID and a district support partner to the Department of Health (DoH) implementing HIV, tuberculosis and sexually transmitted infection programs in Johannesburg, Cape Town, Sedibeng, Mopani and Capricorn Districts. Anova supports the public health care system by providing technical assistance and direct service delivery. In order to implement PNC, Anova employed nurses and lay health providers to organise, recruit for and run the clubs. All staff were trained on the PNC model using materials provided by MSF.

Anova and the Johannesburg Health District Department of Health established PNC at 12 public primary healthcare facilities in Johannesburg from July 2019 to June 2020 and the study was conducted in all these facilities.

\section{Study design}

We used semi-structured in-depth interviews (IDIs) to collect data from health care providers in Anova supported health facilities. We adapted semi-structured interview guides from the RE-AIM Qualitative Evaluation for Systematic Translation (RE-AIM QuEST mixed methods framework) (17). The framework 
proposes open-ended questions in each RE-AIM dimension that are applicable across interventions, study types, and settings. Table 1 shows the RE-AIM dimension definitions and adapted key questions from which the study interview guides were developed.

A researcher invited all PNC facilitating health providers (20) in the twelve PNC-implementing health facilities to participate in interviews. These health providers were specifically hired and trained by Anova to support DoH in PNC implementation.

We collected data in May and June 2020. A researcher (AJ) with experience in qualitative data collection conducted interviews telephonically using the semi-structured interview guide. Interviews took between 40 to 80 minutes. All interviews were audio-recorded and later transcribed verbatim. Transcription was performed by the researcher who conducted the interviews. Transcripts were not returned to the participants for comment or correction

Table 1: Implementation outcomes- RE-AIM dimensions and key questions about postnatal clubs addressed during data collection and analysis 


\begin{tabular}{|c|c|c|}
\hline $\begin{array}{l}\text { RE-AIM } \\
\text { Dimension }\end{array}$ & Definition & Key questions addressed \\
\hline Reach & $\begin{array}{l}\text { Is the } \\
\text { intervention } \\
\text { reaching the target } \\
\text { population? }\end{array}$ & $\begin{array}{l}\text { What are the barriers and facilitators to PNC enrolment, } \\
\text { and how can they be addressed? } \\
\text { What are the barriers and facilitators to participation for } \\
\text { clients? }\end{array}$ \\
\hline Effectiveness & $\begin{array}{l}\text { Does the } \\
\text { intervention } \\
\text { accomplish its } \\
\text { goals? }\end{array}$ & $\begin{array}{l}\text { What are health providers' perceptions on whether PNC } \\
\text { is achieving the same patient outcomes and having the } \\
\text { same impact as in the original design? } \\
\text { What are the conditions and mechanisms that lead to } \\
\text { effectiveness? }\end{array}$ \\
\hline Adoption & $\begin{array}{l}\quad \text { To what extent } \\
\text { are those targeted } \\
\text { to deliver the } \\
\text { intervention } \\
\text { participating? }\end{array}$ & $\begin{array}{l}\text { What affects health provider participation? } \\
\text { What do health providers like about the intervention? } \\
\text { intervention to prevent or encourage adoption? Was there } \\
\text { partial or complete adoption? Why did some health } \\
\text { providers in these facilities participate and others did not? }\end{array}$ \\
\hline Implementation & $\begin{array}{l}\quad \text { To what extent } \\
\text { was the } \\
\text { intervention } \\
\text { consistently } \\
\text { implemented }\end{array}$ & $\begin{array}{l}\text { What were the modifications to the intervention and why } \\
\text { did they occur? } \\
\text { What were the barriers to fidelity? } \\
\text { What are the contextual factors and processes' } \\
\text { underlying barriers to implementation and how do we } \\
\text { address them? }\end{array}$ \\
\hline Maintenance & $\begin{array}{l}\text { Extent to } \\
\text { which the program } \\
\text { has been } \\
\text { institutionalised }\end{array}$ & What are the barriers to continuing the program? \\
\hline
\end{tabular}

Note: Adapted from RE-AIM QuEST mixed methods framework

\section{Analysis and interpretation}

The electronic transcripts were loaded into Nvivo 12 qualitative data software for analysis. We utilised a multistage analytical strategy to identify key themes, codes, and sub codes(18). In the first stage, a researcher (NMZ) prepared an initial list of parent codes and definitions based on the study objectives, interview guide, and existing literature on group postnatal care. Examples of parent codes included: group sessions implementation, resource constraints, and psychosocial support and training. The same researcher applied these parent codes using four transcripts, in the process identifying more parent codes and relevant sub codes under each parent code. For example, under the group sessions implementation 
parent code we added the following sub codes: client-initiated discussions, topic guided discussions, group composition and confidentiality. In this way a preliminary codebook, which included both predetermined and emergent codes was developed. Then, all the remaining transcripts were coded using this preliminary codebook. Data were indexed by identifying segments of the data that corresponded to a particular code. Using thematic analysis (19), we analysed the coded transcripts using the key questions under each of the RE-AIM dimensions described in Table 1 above. All codes and themes were reviewed independently by another author (AJ).

\section{Ethical consideration}

The Human Science Research Council (HSRC) research ethics committee (REC) approved the study, $3 / 22 / 08 / 18$. We obtained informed consent from participants prior to the commencement of the interviews. The research facilitator explained the purpose of the study prior to data collection. Participants consented to the use of a digital audio recorder. The transcripts and recordings were kept in password protected devices.

\section{Results}

In total 15 interviews were conducted with Anova employed health care providers (9 lay health providers and 6 nurses). The health providers were interviewed based on availability and willingness to participate in the data collection. Interviews were conducted in 12 primary health facilities.

In order to provide context to the findings, respondents were asked to describe the duties which they conducted as part of their roles. The majority of the lay health providers described their role as that of a PNC facilitator, indicating that they are responsible for preparing and running club sessions. This included recruiting MIPs, preparing meeting and consultation rooms, ordering medication, psychosocial support, conducting peer support, recording club visits in the register, distribution of pre-packed medication, flagging high viral loads and positive HIV test results to the PNC nurse and tracing flagged high viral load and positive HIV test clients.

The nurses reported that they were responsible for clinical oversight of PNCs. Activities included provision of drug scripts for club members, provision of clinical care (HIV and non-HIV) for mothers and infants (general child health and PMTCT), blood tests (viral load, HIV tests), conducting pap smears and providing enhanced care for high-risk mothers.

Participants reported that they provided services and identified a range of benefits, challenges and suggestions; these are discussed below. The results are presented below within the dimensions of the REAlM framework.

Reach

The reach dimension included investigating participants' perception of clients' willingness to participate in PNC. Key topics included enablers and barriers related to the program, with a particular focus on 
enrolment strategies. A key facilitator to the reach of the program was the provision of integrated care through the "one-stop-shop" model. Health providers cited that the model was preferable to clients, as it offered convenient medication pick-up processes and reduced waiting times and clinic visits.

Furthermore, participants indicated that the model supports HIV status disclosure to families and provides clients with a space to form cohesive and positive group dynamics over time, thereby contributing to adherence to ART.

they do like the model because they have built relationships with everyone, so we engage together, they like the whole setting of sitting together and seeing each other on the same appointment day

PNC counsellors and nurses also cited that they were able to support clients beyond club days. Providers reported communicating through WhatsApp (either over groups or individually) which was also mentioned by providers as facilitating willingness of clients to participate in the model.

And also one other thing that they appreciate is this WhatsApp group that we have created because we can communicate with them to ask them to come to the clinic

In turn, participants noted that the main reason clients declined to use the program was fear of unintended disclosure. They described how some clients avoid going to clinics nearest to them or participating in HIV care because they did not want to be seen at the clinic collecting ART medication or participating in adherence clubs and, in doing so, being linked to having an HIV positive status. Another reported barrier to enrolment was mothers' perceived lack of importance of continued care postpartum. This was particularly seen in multiparous women.

\section{Effectiveness}

Our assessment indicated that providers perceived PNC attendance to be associated with a considerable increase in retention in care. Participants further indicated that the model was effective in improving client adherence to medication.

Firstly, before we had the postnatal club, we used to have defaulters because they were waiting in the queue for a long time, waiting for medication and then the next day they have to come for family planning and then the following day they have to bring the child in for immunization and that takes a lot of time. After starting the Postnatal clubs, we see cooperation because the mother does not default anymore.

Additionally, providers pointed out that PNC contributed to decongestion of health facilities, with MIPs able to receive ART care and child health services in a single clinic visit, decreasing the number of clinic consultations and visits that take place overall. Furthermore, participants indicated that the model provided enhanced care for MIPs. 
But now with the PNC, we dig deeper, we check the mother('s) viral load, we check if they are taking the medication, we engage with the mothers unlike when I used to do it before. PNC makes more sense than what I used to do before

Providers believed the model has a potential in improving MIPs outcomes, however, many participants recognized that availability of resources for proper implementation ultimately determines the effectiveness of the intervention.

\section{Adoption}

The South African National Department of Health recommends the use of differentiated modalities of care (DMoC) nationwide to encourage adherence and retention in care, with PNC being a form of DMoC for postnatal women and their infants. All participating providers expressed support for the PNC model and a belief that the program has potential to provide efficient, comprehensive and caring services. The assessment found consensus among participants that efforts towards scaling up PNC were perceived as worthwhile. Participants indicated the conventional PMTCT postnatal services, where mothers receive care independently from their infants, and HIV care may also be provided separately, do not cater to all maternal and infant needs. In their view, PNC was designed to address the specific needs and expectations of MIPs.

To ensure that the PNC model was implemented in a standardized format across the district, all Anova staff who were selected to work on PNC received standardized training on the model and were given manuals and job aids. A majority of the participants indicated that they had received this training and that it assisted them with implementing the model.

the workshop that I have mentioned, helped us a lot in a sense that by the time we got to the facility we were prepared with and we knew what is expected from us and what we are dealing with and how to implement it.

However, most participants noted a lack of appreciation of facility staff in the model, with them not directly implementing postnatal clubs and questioning participants about the value of the PNC model. They reported that facility staff perceive that the intervention was developed for and implemented by an external entity;

Because when you come in they would say "no this is an Anova thing

Despite facility staff's lack of involvement, some participants reported pockets of support.

Firstly the management gave us all the support that we needed in terms of using their staff to attend to our patients on Fridays and again when we got here the two mentor mother's walked us through what happens on Fridays to conduct the club's because they had their experience .... on how to run the club's so they took us to step by step from getting files and preparing 
Another participant reported collaboration with other non-PNC implementing staff:

Because we understand each other, if she sees someone even on her side (work station) who is not the part of the club but needs the intervention, she knows where to direct that person and she knows how to assist that person and she knows what I am here for.

Participants cited that involvement of government department stakeholders was necessary to promote their buy-in and support the integration of the intervention within established workflows of the clinics.

Participants suggested that demonstrating the competitive advantages of PNC with improved communication and dissemination would be beneficial, i.e., showcasing that the model was designed to make a difference. Participants emphasized the need to support other facility staff not directly involved in implementing the clubs to understand what role or gap PNC is designed to fill.

Implementation

While the PNC model has proven to be acceptable to our study participants, the implementation of the model is not without challenges. As described above (Figure 1), PNCs have four core components which encourage the provision of structured, standardized care. However, participants reported variations in implementation that they attributed largely to a lack of resources. One major challenge cited by many providers was unavailability of space to conduct group sessions. The concern about space was repeated by several participants, this challenge was linked to deviation from the group model.

if we can have space where we will do the sessions. Because now we call this PNC but we see mothers one by one and they do not know that they need to be in a group with all the other mothers.

Inadequate human resource was also identified as a constraint to effective delivery of PNC in the facilities. Health providers reported that PNC are inadequately staffed, emphasizing the shortage of professional nurses, which resulted in nurses rotating among different facilities rather than being able to focus on PNC implementation at a single site. This had led to some facilities deviating from the model by conducting groups with different infant age categories in a single group. In the original PNC model, MIPs are grouped according to the age of the baby.

Other providers felt that appropriate planning prior to implementation should have taken place to ensure the necessary resources (e.g., space, staffing and equipment) were in place prior to the start of the project.

I think before the Postnatal club was implemented in September, it would have worked if we identified the space where the session will take place. Because what happened is that we went to the facility, we told them about the PNC and we started and no resources were identified for us so, we had to arrange for ourselves to see how we go about.

Other factors that constrained the implementation of PNC included insufficient medical equipment and lack of communication gadgets like phones Participants reported that access to a "one-stop-shop" model 
was not possible in some facilities because of a lack of essential equipment, which meant clients had to move around the facility to access other services that cannot be provided in the same room.

The baby scale [is a challenge] and I also struggle with the BP scale [blood pressure monitor] to check the blood pressure for the mothers. I do not have a weight scale. Sometimes it gets confusing for them because I tell them that we will do everything in one room, now I don't do that. so, they need to go on the other side to do their weight and come back to me, so, those are my challenges.

There were concerns around suboptimal implementation of clubs with some PNC components missing. Specifically, participants reported that there were no health talks, very few group meetings and clients were not accessing care in one room. This was cited by some providers to have contributed to a lack of adoption, likely because the model appears more cumbersome and less efficient than it was originally designed to be. Participants pointed out that the current state of implementation in some facilities was perceived by other staff as a duplication of existing immunization and PMTCT programs.

So, there is no space to sit together and have a session so we both do it individually, so that is why I am saying it is a duplicate [of] what is being done on the other side of the clinic...... if I invite the sister to come and join us for the session, she would ask me what are you doing there that is different from what I do? and I would not be able to answer her because there is nothing that is different, so I do not see that there is a gap that is being filled.

Even though participants reported challenges in the implementation of the PNC, all participants indicated that they had to adapt the model to ensure the intervention was delivered. This flexibility is necessary as PNCs need to integrate with the health facility's processes in order to be successful. This emphasizes the importance of maintaining agility when implementing PNC at health facilities.

\section{Sustainability}

Sustainability includes both integration of an intervention into routine practice and integration of a limited number of program components that may improve patient care (19). The integration of an intervention into routine practice has been linked with prospects for maintaining the intervention. A key driver of successful institutionalization at the facility level was having the necessary resources readily stocked and available, which requires support from and coordination with facility management. Participants believed that the project would likely not be sustainable in the future due to the lack of support and adoption of the model by the non-PNC focused facility staff.

That is why there is no support at all because they feel that its ..... not a national mandate.

When asked what would facilitate continuing the project, participants felt that this will require increased advocacy support from facility management.

\section{Discussion And Recommendations}


The goal of this qualitative study was to understand factors influencing PNC implementation from health providers' perspectives. Health providers generally perceived PNC to have many benefits to mothers and their infants; however, they felt that a lack of resources (e.g., space, medical equipment, staff) negatively impacted reach and sustainability. Study participants reported variable implementation of PNC components due to the lack of resources, for example mothers were counselled individually instead of in groups.

Our findings suggest that participants perceived benefits of the intervention were selling points when recruiting potential clients, consequently facilitating intervention reach. This study found that the PNC model is perceived by participants to have the potential to enhance maternal retention in care and adherence to ART and prevent MTCT in the postnatal period. Results revealed barriers to reach, for example some clients believed additional support during the postnatal period is unnecessary. Other clients were reported to recognize the benefits of the model, but encountered a range of barriers to participation including fear of unintended disclosure. An implementation science systematic review by Mukubanga et al on barriers and facilitators to enrolment and participation in adherence clubs (from which PNC is adapted) identified similar barriers to reach or enrolment (20).

Our study described similar perceptions of effectiveness as previous studies on implementation of adherence clubs and postnatal groups $(21,22)$. Adherence clubs were highly acceptable to stakeholders, given the observed benefits, including decongestion of clinics, increased social support for clients and the low cost of implementation (23). An MSF Project report showed that PNC have good early retention in care of the MIP and viral load suppression, as well as zero HIV seroconversion in the infants in similar setting (24). Another study showed that the PNC model supports knowledge acquisition, triggers behavioural change, allows for peer support and satisfaction from the perspective of PNC clients, staff and key informants (25).

PNC were acceptable to PNC facilitating staff; however, the model is not well adopted by other non-PNC dedicated staff, and the management of the clubs can also be challenging. Our findings indicate that several facility-level factors hindered greater adoption of the intervention, including limited physical space for program delivery and low ownership by management and non-PNC staff who perceived the model to be duplication of the current standard of care. Adoption depends on the commitment of facility health teams and their understanding of the benefits of the model (8). Many participants suggested increasing communication between stakeholders and fostering a culture of learning. As previously reported, communication can highlight positive experiences of providers and encourage clients to advocate for an intervention (4).

In general, we found inconsistent implementation of the four components of the model attributed to lack of resources highlighting the need to further interrogate the model to identify key components or combinations of components that could lead to similar benefits in terms of retention and viral suppression. The PNC model, which is based on the adult adherence club model, does allow for adaptions to different contexts, usually based on existing resources. A process evaluation of adherence 
clubs reported that different regions in South Africa vary in their approaches to implementing adherence clubs (26). PNC have certain core components for the purpose of providing a structured program of implementation. For example, PNC requires that providers conduct group meetings and offer some level of "one-stop-shop" care to clients. Our study has shown that most facilities lack space and other resources, which made "one-stop-shop" care difficult and sometimes impossible. In these facilities, clubs would run without the group support component or care may be provided in different rooms of the clinic. It is not yet known whether PNC without the group or "one-stop-shop" components would lead to similar benefits in terms of retention and viral suppression in mothers. While an adaption of the "one-stop-shop" may mean that all care is not provided in the same room, it may encourage better clinician communication, a reduction in waiting in queues at each service point, and lead to all care happening on the same day. Further evaluations could explore if the model as it exists is optimal and efficient and whether all four components are needed to ensure similar outcomes.

Sustainability is a limitation of many programs (27) Previous assessments of the model reported concerns around sustainability due to the perception that it increased the workload for staff involved. Similar to our results, stakeholders relevant to implementation of the model into real world practice reported that the expansion of the PNC model could be jeopardized by human resource constraints attributed to strong dependency on externally-funded staff and minimal involvement of non-PNC focused staff (21). Lessons learned from other differentiated models of care provide suggestions on ways to ensure sustainability of the PNCs, including task shifting of some tasks, such as counselling, drug dispensing and administrative tasks, which may be delegated to PNC graduates or PNC members(20).

Use of the RE-AIM framework allowed us to systematically identify facilitators, challenges, opportunities and lessons learned, as related to reach, adoption, fidelity and sustainability, to be used in further implementation of PNC. While there has been a shift towards the integration of ART into routine postdelivery care, including differentiated care $(8,28)$ PNCs are yet to be scaled up in SA. Provider experiences from this study demonstrate that successful integration requires a health system-wide commitment at both planning and implementation stages $(5,29)$. The RE-AIM framework has been used to provide a systematic way to engage service providers to refine interventions to fit within the intervention context. For example, Hacket et al used the implementation outcomes framework to assess the implementation of a Postpartum Contraception Initiative in Tanzania to enhance the understanding of the implementation process, revealing the implementation dynamics, and highlighting potential entry points for improvement in program delivery(30). The study found that providers were enthusiastic and receptive to the initiative. Similar to our study, they found that health system and resource constraints made adoption and fidelity to the intended intervention challenging. Parallel to our findings, many providers questioned the sustainability of the initiative, and most agreed that changes to the initiative's design (e.g., improved staffing, and availability of resources) would strengthen future iterations.

Similarly, Forman and colleagues applied the RE-AIM QuEST framework for both real-time and retrospective evaluation in a pragmatic cluster randomized controlled trial of the Adherence and Intensification of Medications (AIM) program(31). Using the framework, the researchers expanded 
retrospective evaluation of effectiveness by examining why the intervention worked or failed to work and explained which components of the intervention may have been barriers. Similarly, the framework helped our study to identify the group component of PNC as an implementation barrier.

This qualitative study has helped highlight that both pre and post intervention implementation stages are important to the success of intervention. Table 2 below summarizes recommendations categorized in phases from planning to implementation.

Table 2

Recommendations for planning and implementation of PNC

\begin{tabular}{|ll|}
\hline Stage & \\
\hline Planning & $\begin{array}{l}\text {-Support facility managers and existing staff to implement PNC, including facility } \\
\text { staff who will collaborate with implementation } \\
\text { - Assess and allocate available physical resources, such as consultation and } \\
\text { group space }\end{array}$ \\
\hline $\begin{array}{l}\text { Pre- } \\
\text { implementation }\end{array}$ & $\begin{array}{l}\text { - Training and sensitization of the implementing and other staff who collaborate in } \\
\text { the implementation is important }\end{array}$ \\
\hline Implementation & $\begin{array}{l}\text { - Increasing communication between stakeholders and fostering a culture of } \\
\text { learning among stakeholders }\end{array}$ \\
& $\begin{array}{l}\text { - Regular interactions with stakeholders throughout implementation to monitor } \\
\text { and adapt the intervention as needed }\end{array}$ \\
\hline Scale Up & $\begin{array}{l}\text { Further evaluation to explore if the model as it exists is optimal and efficient and } \\
\text { whether all four components are needed to ensure similar outcomes. This could } \\
\text { support identification of the core components of the intervention }\end{array}$ \\
\hline
\end{tabular}

Table 2: Recommendations for planning and implementation of PNC

\section{Limitation}

Data collection did not involve non-Anova health providers or PNC clients, so the study team did not have the opportunity to triangulate our results with these two groups. These groups could have taught us more about the barriers and facilitators to reach, adoption and sustainability of the project.

\section{Conclusion}

At this early stage of implementation, our study found incomplete implementation of PNC in many of the participating facilities, mostly due to a lack of resources and stakeholder involvement. This underscores the need for increased support at management level to ensure model efficacy and sustainability. The assessment also highlights the importance of exploring resource availability at the organisation level in order to tailor elements of the intervention to fit within available resources. This study has shown that the PNC model can be implemented with flexibility and should be adapted based on existing resources. 
Our detailed report of the implementation outcomes assessment using the RE-AIM framework offers practical information that can be used for future design and scale-up of PNC.

\section{Abbreviations}

DMoC

Differentiated Modalities of Care

$\mathrm{DoH}$

Department of Health

ART

Antiretroviral Therapy

ECD

Early Childhood Development

HSRC

Human Science Research Council

$\mathrm{MCH}$

Maternal and Child Health

MIPS

Mother-Infant Pairs

MSF

Médecins Sans Frontières

PEPFAR

President's Emergency Plan for AIDS Relief

PLHIV

People Living with HIV

PMTCT

Prevention of Mother-To-Child Transmission Of HIV

PNC

Postnatal Club

RE-AIM

Reach, Adoption, Implementation, Maintenance

RE-AIM QuEST

RE-AIM Qualitative Evaluation For Systematic Translation

REC

Research Ethics Committee

\section{Declarations}

Acknowledgements 
We gratefully acknowledge health providers who took their valuable time to participate in this study. The authors would also like to acknowledge Johannesburg Health district and MSF for their support of the study.

\section{Availability of data and materials}

The dataset (which includes individual transcripts) is not publicly available due to the confidential nature of interview data.

\section{Authors' contributions}

The authors confirm contribution to the paper as follows: NM, KR and JD contributed to the study conception and design; AJ carried out data collection and contributed to analysis; NM conducted analysis and interpretation of result; NM prepared the initial draft of the paper, to which KR, HS, JM, BS and JD revised critically for important intellectual content. All authors reviewed and approved the final version of the manuscript.

\section{Ethics approval and consent to participate}

The study received ethical approval from HSRC REC (3/22/08/18) and the Johannesburg District Research Committee.

\section{Consent for publication}

All participants provided written informed consent before participating in the study, which included consent to publish anonymous quotes from individual participants.

\section{Competing interests}

The authors declare that they have no competing interests.

\section{Author details}

1 Anova Health Institute

2 Division of Community Pediatrics, School of Public Health, University of Witwatersrand

3 Department of Health Behavior, University of North Carolina

4 Division of Infectious Diseases and HIV Medicine, Department of Medicine, University of Cape Town, South Africa

5 School of Public Health and Family Medicine, University of Cape Town, South Africa

6 Department of Community Health, School of Public Health, University of Witwatersrand 


\section{Funding}

This study forms part of Anova Health Institute's technical support funded by the US President's Emergency Plan for AIDS Relief (PEPFAR) through the United States Agency for International Development (USAID) under Cooperative Agreement number 674-A-12-00015.

\section{References}

1. Bhardwaj S, Barron P, Pillay Y, Treger-Slavin L, Robinson P, Goga A, et al. Elimination of mother-tochild transmission of HIV in South Africa: rapid scale-up using quality improvement. S Afr Med J. 2014;104(3 Suppl 1):239-43.

2. Unaids. Start Free Stay Free AIDS Free-2017 progress report. Switzerland: UNAIDS Geneva; 2017.

3. Burton R, Giddy J, Stinson K. Prevention of mother-to-child transmission in South Africa: an everchanging landscape. Obstetric medicine. 2015;8(1):5-12.

4. Myer L, Phillips TK, Zerbe A, Brittain K, Lesosky M, Hsiao N-Y, et al. Integration of postpartum healthcare services for HIV-infected women and their infants in South Africa: a randomised controlled trial. PLoS Med. 2018;15(3):e1002547.

5. Mutemwa R, Mayhew S, Colombini M, Busza J, Kivunaga J, Ndwiga C. Experiences of health care providers with integrated HIV and reproductive health services in Kenya: a qualitative study. BMC Health Serv Res. 2013;13(1):1-10.

6. Duvivier H, Decroo T, Cassidy T, Mbakaza Z, Nelson A, Duran LT, et al. "They share more than we think they are sharing": a mixed-methods evaluation of post-natal clubs in South Africa. F1000Research. $2018 ; 7$.

7. Duvivier H, Decroo T, Nelson A, Cassidy T, Mbakaz Z, Duran LT, et al. Knowledge transmission, peer support, behaviour change and satisfaction in post Natal clubs in Khayelitsha, South Africa: a qualitative study. Reproductive Health. 2020;17(1):1-11.

8. Trafford Z, Gomba Y, Colvin CJ, lyun VO, Phillips TK, Brittain K, et al. Experiences of HIV-positive postpartum women and health workers involved with community-based antiretroviral therapy adherence clubs in Cape Town, South Africa. BMC Public Health. 2018;18(1):1-13.

9. Zerbe A, Brittain K, Phillips TK, Iyun VO, Allerton J, Nofemela A, et al. Community-based adherence clubs for postpartum women on antiretroviral therapy (ART) in Cape Town, South Africa: a pilot study. BMC Health Services Research. 2020;20(1):1-12.

10. Zotti ME, Pringle J, Stuart G, Boyd WA, Brantley D, de Ravello L. Integrating HIV prevention in reproductive health settings. Journal of Public Health Management Practice. 2010;16(6):512-20.

11. Bauer MS, Kirchner J. Implementation science: what is it and why should I care? Psychiatry research. 2020;283:112376.

12. Proctor E, Silmere H, Raghavan R, Hovmand P, Aarons G, Bunger A, et al. Outcomes for Implementation Research: Conceptual Distinctions, Measurement Challenges, and Research Agenda. Administration Policy in Mental Health Mental Health Services Research. 2010;38(2):65-76. 
13. Shelton RC, Chambers DA, Glasgow RE. An extension of RE-AIM to enhance sustainability: addressing dynamic context and promoting health equity over time. Frontiers in public health. 2020;8:134.

14. Holtrop JS, Rabin BA, Glasgow RE. Qualitative approaches to use of the RE-AIM framework: rationale and methods. BMC Health Serv Res. 2018;18(1):177.

15. Glasgow RE, Harden SM, Gaglio B, Rabin B, Smith ML, Porter GC, et al. RE-AIM planning and evaluation framework: adapting to new science and practice with a 20-year review. Frontiers in public health. 2019;7:64.

16. Kganakga C, Munthree C, Mohideen F, Wijk Ev, Massawe D, Nel D, et al. Ending aids in Johannesburg: an analysis of the status and scale-up towards HIV treatment and prevention targets. The World Bank; 2016.

17. Forman J, Heisler M, Damschroder LJ, Kaselitz E, Kerr EA. Development and application of the REAIM QuEST mixed methods framework for program evaluation. Prev Med Rep. 2017;6:322-8.

18. Saldana J. The coding manual for qualitative researchers 2009.

19. Walugembe DR, Sibbald S, Le Ber MJ, Kothari A. Sustainability of public health interventions: where are the gaps? Health research policy systems. 2019;17(1):1-7.

20. Mukumbang FC, Orth Z, van Wyk B. What do the implementation outcome variables tell us about the scaling-up of the antiretroviral treatment adherence clubs in South Africa? A document review. Health Res Policy Syst. 2019;17(1):28.

21. Mangoejane L, Ramukumba MM. Implementation of postnatal care for HIV-positive mothers in the Free State: Nurses' perspectives. African Journal of Primary Health Care Family Medicine. 2019;11(1):1-8.

22. Wilkinson LS. ART adherence clubs: a long-term retention strategy for clinically stable patients receiving antiretroviral therapy. Southern African Journal of HIV Medicine. 2013;14(2):48-50.

23. Dudhia R, Kagee A. Experiences of participating in an antiretroviral treatment adherence club. Psychology health medicine. 2015;20(4):488-94.

24. Nelson A, Duran LT, Cassidy T, Buchanan K, Avezedo V, Abrahams S, editors. Post natal integrated clubs as a way to improve retention in care of mother infant pairs in a primary care setting, Khayelitsha, South Africa2017.

25. Babiker A, El Husseini M, Al Nemri A, Al Frayh A, Al Juryyan N, Faki MO, et al. Health care professional development: Working as a team to improve patient care. Sudanese journal of paediatrics. 2014;14(2):9.

26. McCormack L, Sheridan S, Lewis M, Boudewyns V, Melvin CL, Kistler C, et al. Communication and dissemination strategies to facilitate the use of health-related evidence. Evidence report/technology assessment. 2013(213):1-520.

27. Grimsrud A, Lesosky M, Kalombo C, Bekker L-G, Myer L. Implementation and operational research: community-based adherence clubs for the management of stable antiretroviral therapy patients in 
Cape Town, South Africa: a cohort study. JAIDS Journal of Acquired Immune Deficiency Syndromes. 2016;71(1):e16-23.

28. World Health $\mathrm{O}$. Key considerations for differentiated antiretroviral therapy delivery for specific populations: children, adolescents, pregnant and breastfeeding women and key populations. World Health Organization; 2017.

29. Oliff $M$, Mayaud $P$, Brugha $R$, Semakafu AM. Integrating reproductive health services in a reforming health sector: the case of Tanzania. Reprod Health Matters. 2003;11(21):37-48.

30. Hackett K, Huber-Krum S, Francis JM, Senderowicz L, Pearson E, Siril H, Ulenga N, Shah I. Evaluating the Implementation of an Intervention to Improve Postpartum Contraception in Tanzania: A Qualitative Study of Provider and Client Perspectives. Glob Health Sci Pract. 2020 Jun 30;8(2):270289. doi: 10.9745/GHSP-D-19-00365. PMID: 32606094; PMCID: PMC7326523.

31. Dudley L, Garner P. Strategies for integrating primary health services in low-and middle-income countries at the point of delivery. Cochrane database of systematic reviews. 2011(7).

\section{Figures}

\section{Each PNC session features four key components:}
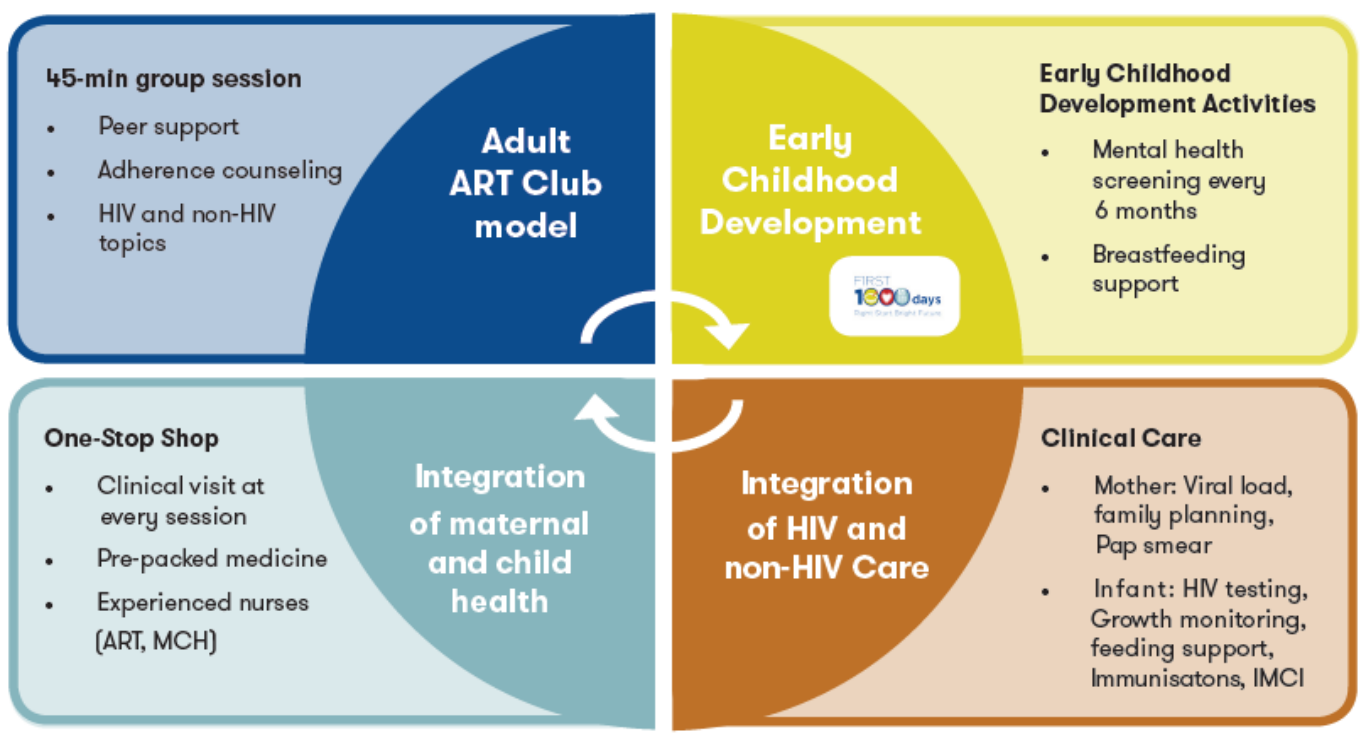

$\mathrm{VL}=$ Viral Load

$\mathrm{FP}=$ Family Planning

$\mathrm{ART}=$ antiretroviral therapy

$\mathrm{IMCl}=$ intergrated management of childhood illness

\section{PNC timeline}

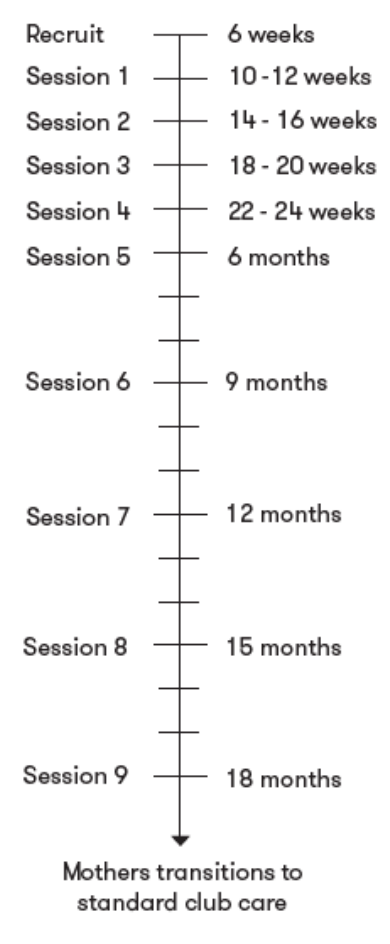

\section{Figure 1}

\section{[7]: PNC components}


[7] Figure from "Duvivier, H., Decroo, T., Nelson, A. et al. Knowledge transmission, peer support, behavior change and satisfaction in post Natal clubs in Khayelitsha, South Africa: a qualitative study. Reprod Health 17, 107 (2020). https://doi.org/10.1186/s12978-020-00957-0"

\section{Supplementary Files}

This is a list of supplementary files associated with this preprint. Click to download.

- AppendixCOREQChecklist.docx 